\title{
Effects of shortwave spectrum on phototaxis trapping of agricultural pests and its optimizing construct as pests-trapping lamp*
}

\author{
Jian Zhou \\ School of Arts \& Science, Sias International University, \\ Xinzheng, Henan431150, China \\ E-mail:master505@sina.com \\ www.sias.edu.cn \\ Qiang Zhou ${ }^{\dagger}$ and Jian-Ping $\mathrm{Li}$ \\ College of Engineering, China Agricultural University, \\ Beijing 100083, China \\ ${ }^{\dagger}$ E-mail: zq@cau.edu.cn \\ www.cau.edu.cn
}

\begin{abstract}
Shortwave spectrum is a main wave band of phototactic trapping agricultural insect-pests Based on the phototactic sensitivity of agricultural insect pests, the shortwave spectrum character of purple, blue and green LEDs is analyzed by means of the fiber optical spectro-meter, and the compsite LEDs as the trapping light of insect pests are constitued by modifying the proportion of purple, blue, and green in the composite LED light. The phototactic trapping effect of insect pests reaches a optimizing level when the proportion of purple, blue and green LEDs is 2:8:4 in the light. Utilizing the sectional type and straight line type manners, the composite LED lights of two constructed types are fabricated, further the pest-trapping lamp is constituted by assembling electrical parts and mechanical units.. The phototactic trapping test of pests in the orchard verify the effectivity of composite LED light in the control of agricultural pests.
\end{abstract}

Keywords: Shortwave Spectrum; Phototaxis; Agricultural Pests; Pest-trapping Lamp; Optimizing Construct; Pests Control; Composite LED light.

\section{Introduction}

Shortwave light is refer to green, blue and purple lights. It possess a higher photon energy than long-wave light, hence has a larger ability to induce visible stimulus for living organism. We know that the blue and purple lights are two key light components taking part in plant photosynthesis, and only some of blue

* This work is supported by National Nature Science Fund of China (NSFC 51375482) and the Special Fund for Agro-scientific Research in the Public Interest ( SFAR-PI 201203025).. 
wavelength spectrum can lead to the influence on human optical biorhythm effect[1,2]. Further shortwave light, especially purple, has an obvious effect on the phototaxis trapping of insect pests, such as lepidoptera and coleoptera pests. Bio-photo-electronic effect of insects and its application in agricultural pest control are discussed in some reports[3,4]. Therefore the shortwave light as a manner of controlling agricultural pests without pesticides is not only beneficial to protect the environment around us and to safeguard agricultural product quality, and its trapping pests as biological protein sources can be utilized to feed the poultry to keep the sustainable development of agricultural production[5].

As the development of light emitting diode (LED) technology, the composite of short-wave LEDs is becoming into a main manner to fabricate the pest trapping lamp[6]. In this paper, the spectral components of shortwave light sources suitable for trapping insect pests are analyzed on the basis of insect phototactic character, and some of shortwave light sources constituted by LEDs is verified to be effective in the phototaxis trapping of orchard pests. This supplies a good basis for developing effective pest-trapping lamps. .

\section{Light Source Spectrum for Trapping Agricultural Pests}

\subsection{Light Wave Requirements of Pests Phototaxis}

The phototaxis of agricultural pest stems from the response of insect ommatidium rhabdome on the photons. The differences in the kind of insects will result many different responses of insect-pests phototaxis on the shortwave spectrum. In table 1 the sensitive wavelength of some insects were summarized by analyzing related references.

Table 1 Sensitive wavelength of some pests

\begin{tabular}{|l|l|l|l|}
\hline \multicolumn{2}{|c|}{ Inscet pests } & Sensitive Spectrum \\
\hline \multirow{3}{*}{} & & Snout moths & $445,475,547$ \\
& \multirow{4}{*}{ Lepidoptera } & Cotton bollworm & $363,385,466$ \\
& & Tobacco budworm & $333,385,466$ \\
& & Peach fruit moth & 520 \\
& & Pear fruit moth & 353,530 \\
\cline { 3 - 4 } & \multirow{4}{*}{ Coleoptera } & Agrotis ypsilon & 370,405 \\
& & Anomala corpulenta & 405,465 \\
& & Holotrichia parallela & 405,465 \\
& & Holotrichia parallela & 360,400 \\
\cline { 3 - 4 } & Hemiptera & Green plant bug & $400 \sim 450$ \\
\hline \multirow{2}{*}{ Beneficial } & & Chrysoperla sinica & 380 \\
& & Propylaea Japonica & $340,483,524$ \\
& & Harmonia axyridis & $360,420,524$ \\
\hline
\end{tabular}

It is obvious that the sensitive wavelengths of insect phototaxis are mostly in the blue, purple, even ultro-violet zone, but a main category of plant pest, snout moths, also includes the sensitive wavelength of green light. At same time 
the beneficial insects presents a more broad phototaxis from ultroviolet, purple to blue, even green light.

\subsection{Effect of Composite Light-waves on Pest Phototaxis}

As the development of photon-electronic technology, the mono-chromatic light LEDs, especially SMD LED flexible rope light, were widely used to recombine the complex light sources for some actual application. Figure 1 tests the spectrum characters of 5050 type SMD LED flexible rope lights with purple, blue, and green lights by using AvaSpec- ULS 2048X36 fiber optical spectrometer.

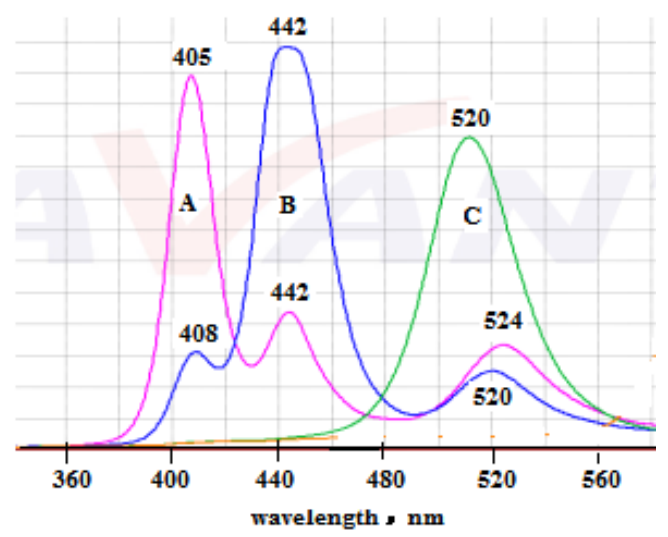

A. Purple light LED; B. Blue light LED; C. Green light LED

Fig. 1. Spectrum characters of SMD LEDs

It can be seen that the emission peaks of purple and blue LEDs present the higher intensity and smaller full width at half maximum(FWHM), at same time, they company with some sub peak wavelengths. The green LED indicates its monochromatic character, but it has a wider FWHM. However the composite light spectrum suitable for trapping agricultural pest listed in table 1 may be recombined by different proportion of purple, blue and green LEDs. Figure 2 presents the trapping effects of different light spectrum proportion on the pests. 


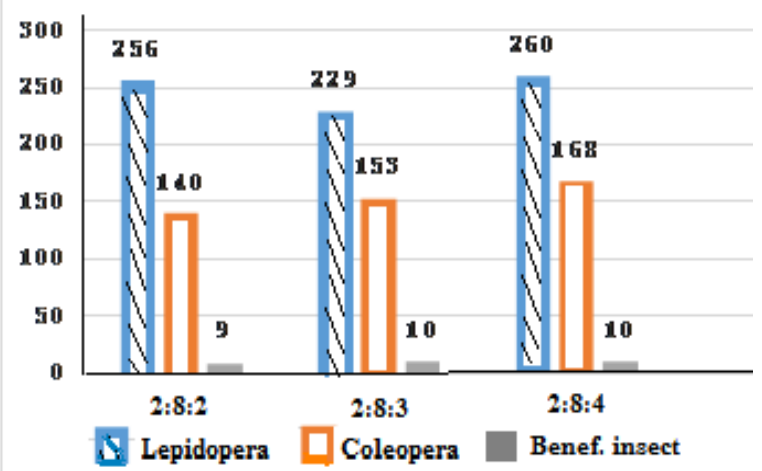

Fig. 2. Trapping effects of different spectrum proportion on pests

When the spectrum ratio of purple to blue was fixed in the ratio of 2:8 as main spectrum for trapping pests, the increase of green light proportion in the total spectrum energy is beneficial to get a better trapping effect on the insect pests. Specially the trapping proportion of composite spectrum on the coleopera pests reaches a maximum level in the green light proportion from 2 to 4 .

\section{LED Light Source Construction for Trapping Agricultural Pests}

\subsection{Configuration of LED Composite Light Source}

The SMD LED flexible rope light can be conveniently used to construct the trapping lights of pests according to LED emitting spectrum and pests' phototactic spectrum. It may twist on the surface of cylindrical PVC tube alternately by different spectrum LED to construct a sectional type LED trapping light as shown in Figure 3A, or stick on the surface of PVC tube along the direction of cylindrical axis alternately by different spectrum LED to structure a straight line type LED trapping light source as shown in Figure 3B.

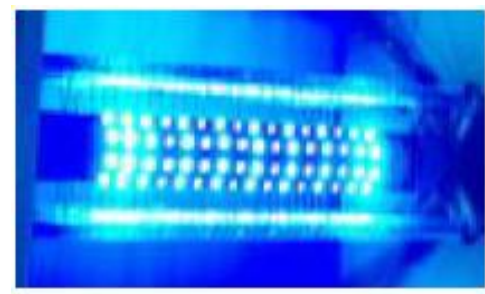

A. Sectional type trapping light

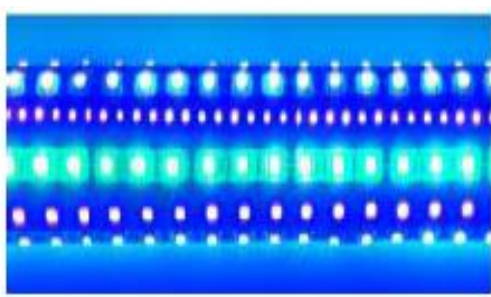

B. Straight line type trapping light Fig. 3 LED trapping light of two constructed types

Regardless of sectional type or straight line type LED trapping light, the uniformity of their emitting light field at the identical radial space spots can be 
identified only when LED flexible ropes distribute reasonably on the cylindrical PVC tube. Figure 4 presents the optimizing spectrum character of trapping light when the spectrum proportion of purple, blue and green is fixed in the 2:8:4. Because the compositing of alike wave peaks, some of sub-wave peaksare fused into three main wave peaks. It enhance the illuminating intensity of three main peaks, and strenthen the linkage of three main wave peaks. Therefore a optimizing spectrum character of composite LED light was obtained.

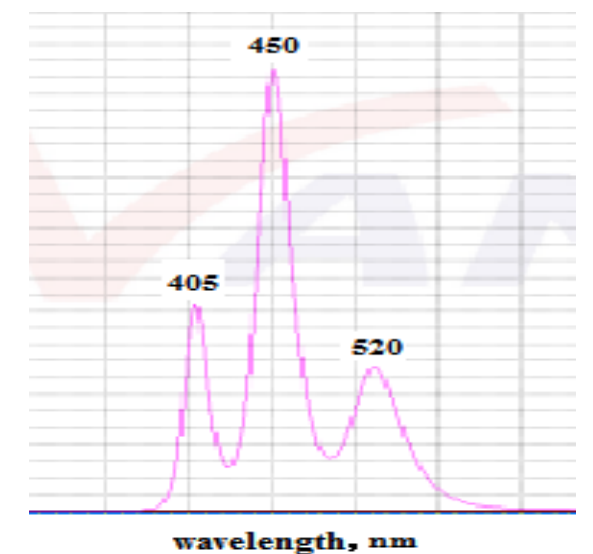

Fig. 4 Spectrum character of composite LED trapping light

Fuether the composite LED intensity of illumination was measured by using the digital illuminometer of model ZDS-10H with the precision of $0.001 \mathrm{Lux}$. At $1.00 \mathrm{~m}$ distance far from LED Light, the purple, blue and green light in the composite LED light keep respectively the illuminating intensity of 1.062, 3.840 and 1.353Lux, and the scope of composite LED spectrum covers the phototactic trapping spectrum of main insect pests.

\subsection{Trapping Test Effects of Composite LED Light in Orchard}

In the test condition of cherry orchard, three zones were selected to set up the composite LED light(2:8:4) to test the phototactic trapping effects to insect pests. At same time, the composite LED light was assembled by a static electricity grid with the high voltage of $2000 \mathrm{v}$ to electrically stunn or kill the insect pests, as show in figure 5 . 


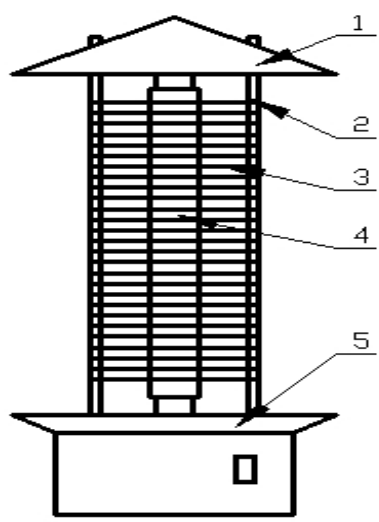

Fig. 5 Schem of composit LED traaping lamp

1.Top cover; 2. Suport post; 3. Electric grid; 4. LED light; 5. Base 2.

Figure 6 indicates the phototactic trapping results of composite LED light on the orchard pests in the densely populated area, ordinary populated zone and its middle strip. It can be seen that the composite LED trapping lamps obtain some of excellent results for insent pests, despite of differences in the zones.

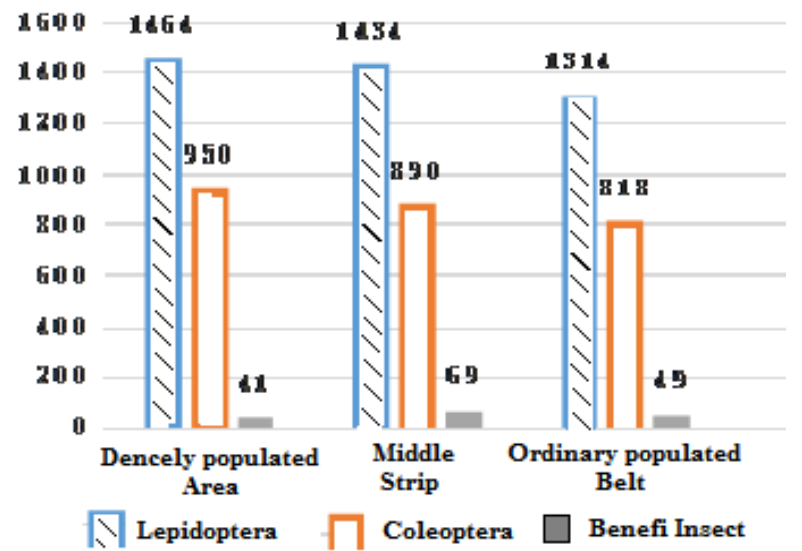

Fig. 6 Trapping test of composite LED light in different zones.

Obviously It is beneficial to trap the mass insect pests hiden in the densely populated area, and the damage to some beneficial insents may be reduced to a minimum level. It indicates that the larger the insect pest density is, the higher the trapping selectivity of composite LED lamp is.

\section{Summary}

The phototactic sensitivity spectrum of insect pests is analyzed and the 
composite LED light with shortwave character are constituted on the base of the requirement of phototactic trapping pests. The 2:8:4 optimizing proportion of purple, blue and green in the composite LED light is verified to be effective to trapping pests.

The single spectrum characters of purple, blue and green LED are tested by means of AvaSpec- ULS 2048X36 fiber optical spectro-meter, and the shortwave spectrum characters of composite LED light are optimized in the 1.063:3.840:1.353 Lux light tensity propotion of purple, blue and green to more adapt for trapping agricultural pests.

The composite LED lamp display a widely adapted results in the phototactic trapping test of orchard pests, especially in the densely populated area. It shows that the composite LED lamp is a more beneficial method to control the mass insect pests hiden in the densely populated area.

\section{References}

1. Han Yu-Hong, Wang Yu-Rong, Jin Shang-Zhong, etc. Influence of Different Wave-length Blue LED on Human Optical Biorhythm Effect. Chinese Journal of Lumi-nescence, 34(2013): 1061-1065. (Ch)

2. Weng Yu-Xiang. The basic principles of under lyingul trafastenergy and electron transfer in primary photosynthesis. Physics, 36(2007):820-832.(Ch)

3. Zhou Qiang, XU Rui-Qing and Cheng Xiao-Tong. Bio-photo-electronic Effect of insects and Its Application in Pest Control. Progress in Modern Bio-medicine, 6(2006): 70-72. (Ch)

4. Roy H.A. van Grunsven, M. Donners, K. Boekee, etc. Spectral composition of light sources and insect phototaxis, with an evaluation of existing spectral response models. Journal of Insect Conservation, 18(2014) :225-231.

5. Wim Verbeke, Thomas Spranghers, Patrick De Clercq, Stefaan De Smet, Benedikt Sas, Mia Eeckhout. Insects in animal feed: Acceptance and its determi-nants among farmers, agriculture sector stakeholders and citizens. Animal Feed Science and Technology, 204(2015): 72-87.

6. Li Bo, Liu Qi-Hang, Zhou Qiang. Investigation of LED insecticidal lamp trapping ochard insect. Agricul- tural Engineering, 3s(2013): 21-25. (CH) 